Zur Biogenese des Leucopterins

\title{
Zusammenhänge zwischen Purin- und Leucopterin-Biogenese in Pieris brassicae $\mathrm{L}$
}

\author{
Von Helmut Simon, Friedrich Weygand, Josef Walter, Hans Wacker \\ und Klaus Schmidt
}

\author{
Aus dem Organisch-chemischen Institut der Technischen Hochschule München \\ (Z. Naturforschg. 18 b, 757-764 [1963] ; eingegangen am 10. Juni 1963)
}

Herrn Professor Dr. Отто Warburg in Verehrung zum 80. Geburtstag

\begin{abstract}
Es werden die spez. Radioaktivitäten von Purinen und Leucopterin aus Kohlweißlingen, denen Glycin- $\left[2{ }^{14} \mathrm{C}\right]$ sowie Amino-imidazol-carboxamid- $\left[4^{-14} \mathrm{C}\right]$ zu verschiedenen Zeiten der Puppenruhe appliziert worden war, verglichen. Aus diesen Ergebnissen wird geschlossen, daß eine Umwandlung der Purine in Leucopterin mit einem Minimum an struktureller Änderung erfolgt. Die hohen Einbauraten für ${ }^{14} \mathrm{C}$-markierte Ribonucleinsäuren, Adenyl- und Guanylsäure bestätigen diese Annahme. Bei Gabe von Ribose- $\left[1^{14} \mathrm{C}\right]$ ergibt sich bei kurzer Verweilzeit eine hohe Einbaurate in Leucopterin. Das Aktivitätsverhältnis von C-8 : C-9 * ist dann 14:86. Der Mechanismus des Úberganges Purin $\rightarrow$ Pterin wird diskutiert. - In den Kohlweißlingen wurde neben den üblichen Purinen noch Isoguanin und 2.8-Dihydroxy-guanin nachgewiesen.
\end{abstract}

Mit Hilfe von Vorläufer-Untersuchungen mit ${ }^{14} \mathrm{C}$ markierten Verbindungen konnten wir an Pieris brassicae L. (großer Kohlweißling) zeigen, daß mit Ausnahme der C-Atome 8 und 9 die einzelnen Kohlenstoffatome des Leucopterins aus denselben Vorläufern stammen wie die entsprechenden Kohlenstoffatome der Purine ${ }^{1}$. Als Vorläufer für die CAtome 8 und 9 dienen, wie später von uns gefunden wurde, Zucker, wie Glucose oder Ribose ${ }^{2,3}$, was uns zu der Hypothese veranlaßte, daß aus dem Guanosin oder der Guanylsäure C-8 eliminiert wird und der Ribosylrest unter Ausbildung des PyrazinRinges, evtl. nach einer A m a d or i-Umlagerung direkt einkondensiert ${ }^{2,4}$. Diese Annahme beruhte hauptsächlich auf den Befunden, daß nach Gabe von Ribose- $\left[1-{ }^{14} \mathrm{C}\right]$ ca. $60 \%$ der insgesamt in C-8 + C-9 fixierten Radioaktivität in C-9 lokalisiert waren und die spez. Radioaktivitäten von Guanin und Adenin nach Applikation von Glucose- $\left[2-{ }^{14} \mathrm{C}\right]$, Glycin$\left[2-{ }^{14} \mathrm{C}\right]$ und Formiat $-{ }^{14} \mathrm{C}$ nahezu gleich mit der von Leukopterin waren, falls man auf die beiden Systemen entsprechenden Kohlenstoffpositionen (C-2, 4, 5 und 6) bezieht ${ }^{4}$.

Bei Gültigkeit dieser Annahmen sollte der Einbau von C-1 der Ribose- $\left[1-{ }^{14} \mathrm{C}\right]$ in das C-9 des Leukopterins im Vergleich zu C-8 stark überwiegen.

* Es wird die sog. deutsche Bezifferung benutzt.

1 F. Weygand u. M. W aldschmidt, Angew. Chem. 67, 328 [1955].

2 F. Weygand, Angew. Chem. 71, 746 [1959].
Das von uns gefundene Verhältnis von $6: 4$ war jedoch nicht sehr ausgeprägt. Dies konnte auf der relativ langen Verweilzeit der Ribose im stark stoffwechselaktiven Puppenkörper beruhen. Wir prüften daher das Verhältnis der Radioaktivität von C-8 zu C-9 in Abhängigkeit von der Applikationszeit der Ribose- $\left[1-{ }^{14} \mathrm{C}\right]$.

Weitere Untersuchungen stellten wir zur Frage des Zusammenhanges zwischen Purin- und PterinBiogenese an. Wenn an der engen Beziehung zwischen der Biogenese von Guanin und Leukopterin auf Grund unserer früheren Befunde auch nicht mehr gezweifelt werden konnte, so war zwischen folgenden drei Möglichkeiten noch nicht mit Sicherheit zu unterscheiden ${ }^{5}$ :

1. Die Biogenesewege von Purinen und Pterinen sind weitgehend identisch und vom unmittelbaren Vorläufer der Purine, dem 5-Amino-imidazolcarboxamidribotid bzw. der Formylverbindung ergibt sich eine Verzweigung zu den Purinen einerseits und zu den Pterinen andererseits.

2. Die Purine werden z. B. während der sog. „Puppenruhe" teilweise wieder abgebaut und die Abbauprodukte werden zu Pterinbiogenese herangezogen.

3. Die Purine werden mit einem Minimum struktureller Änderung in die Pterine umgewandelt.

${ }^{3}$ F. Weygand, H. J. Schliep, H. Simon u. G. Dahms, Angew. Chem. 71, 522 [1959].

${ }^{4}$ F. Weygand, H. Simon, G. Dahms, M. Waldschmidt, H. J. Schliep u. H. Wacker, Angew. Chem. 73, 402 [1961]. 
Die Frage des Überganges Purin $\rightarrow$ Pterin wird seit einigen Jahren auch in zahlreichen anderen Laboratorien bearbeitet und diskutiert. Erstmalig wurde der Utbergang von Purin in ein Pterin in Larven von Xenopus in unsrem Arbeitskreis gezeigt ${ }^{6}$. Bei späteren Arbeiten interessierte diese Frage hauptsächlich im $\mathrm{Zu}$ sammenhang mit der Riboflavin- und Folsäurebiogenese. Den endgültigen Beweis für den Übergang des kompletten Puringerüstes mit Ausnahme von C-8 in den Pyrimidin- und Pyrazinring des Rifloflavins brachte McNutr ${ }^{7}$, durch Verwendung von Adenin ${ }^{15} \mathrm{~N}$ und Xanthin $-{ }^{15} \mathrm{~N},{ }^{14} \mathrm{C}$, nachdem bereits $1954 \mathrm{P}_{\mathrm{LAUT}}{ }^{8}$ auf einen möglichen biogenetischen Zusammenhang zwischen $\mathrm{Pu}$ rinen und Riboflavin hingewiesen hatte. Weiterhin waren es neben McNutt und Forrest ${ }^{9}$, Korte und Mitarb ${ }^{10}$, MAsuda und Mitarbb. ${ }^{11}$ und einige andere Arbeitskreise ${ }^{12-14}$, die den Purin-Pterin-Übergang diskutierten.

Die Verwertung von Purinen bei der Folsäurebiogenese wurde von Aaronson und Rodriguez ${ }^{15}$ sowie von $\mathrm{V}_{\text {IEIRa }}$ und Shaw ${ }^{16}$ festgestellt. Besonders überzeugend sind Versuchsergebnisse von Reynolds und Brown ${ }^{17}$, wonach zellfreie Extrakte von Escherichia coli Guanosin, Guanylsäure und andere Guanosinphosphate in Dihydrofolsäure überführen.

Wir versuchten durch Vergleich der spezifischen Radioaktivitäten von Leukopterin, Guanin und Adenin nach Gabe verschiedener markierter Vorläufer zu entscheiden, welche der drei oben erwähnten Möglichkeiten des Zusammenhanges der Purin- und Leukopterinbiogenese im Kohlweißling zutrifft.

Nachdem wir nach Gabe von Glycin- $\left[2-{ }^{14} \mathrm{C}\right]$, Formiat- ${ }^{14} \mathrm{C}$ und Glucose- $\left[2-{ }^{14} \mathrm{C}\right]$ bereits eine weitgehende Gleichheit der spez. Radioaktivitäten von Guanin und Leukopterin festgestellt hatten, interessierten wir uns für die Zeitabhängigkeit des Radioaktivitäts-Verhältnisses von verschiedenen Purinen und Leukopterin. Hierfür schien uns neben Glycin- $\left[2{ }^{14} \mathrm{C}\right]$ der unmittelbare Purinvorläufer Aminoimidazol-carboxamid besonders günstig. Um schließlich auch noch einen direkten Beweis für den Über-

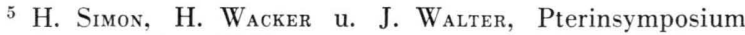
Stuttgart Sept. 1962.

6 I. Ziegler-Günder, H. Simon u. A. W Wcker, Z. Naturforschg. 11 b, 82 [1956].

7 W. S. McNutt, J. Amer. chem. Soc. 83, 2303 [1961].

8 G. W. E. Plaut, J. biol. Chemistry 208, 513 [1954]; s. a. G. W. E. Plaut, J. biol. Chemistry 235, PC 41 [1960].

9 H. S. Forrest u. W. S. McNutt, J. Amer. chem. Soc. 80, 739 [1958].

${ }^{10}$ F. Korte u. G. Ludwig, Liebigs Ann. Chem. 648, 131 [1961] (siehe dort frühere Literatur).

11 T. Kishi, U. Asai, T. Masuda u. S. Kuwada, Chem. a. Pharmaz. Bull. [Japan] 7, Nr. 4 [1959].

12 M. A. Khalididi, Federat. Proc. 17, 180 [1958].

13 E. G. Brown, T. W. Goodwin u. O. T. G. Jones, Biochem. J. 68, 40 [1958]. gang Purin $\rightarrow$ Pterin zu haben, applizierten wir ${ }^{14} \mathrm{C}$ markierte Nucleinsäuren, Ribonucleinsäurehydrolysate, Adenylsäure und Guanylsäure, die völlig oder vorwiegend im Basenteil markiert waren. Schließlich boten wir noch 2.4.5-Triamino-6-hydroxypyrimidin- $\left[2-{ }^{14} \mathrm{C}\right]$ und eine Reihe von Pterinderivaten an.

\section{Methodik}

M arkierte Verbind ungen

Ribose- $\left[1-{ }^{14} \mathrm{C}\right]$ und Glycin- $\left[2-{ }^{14} \mathrm{C}\right]$ waren käuflich erworbene Präparate, die vor ihrer Verwendung papierchromatographisch auf Reinheit geprüft worden waren. 4-Amino-5-imidazolcarboxamid-[4-14 C] wurde ausgehend von Bariumcarbonat ${ }^{14} \mathrm{C}$ synthetisiert ${ }^{18}$. Dabei wurde das Natriumcyanid. ${ }^{14} \mathrm{C}$ durch den Austausch $\mathrm{Ba}^{14} \mathrm{CO}_{3}+$ $\mathrm{KCN} \rightleftarrows \mathrm{BaCO}_{3}+\mathrm{K}^{14} \mathrm{CN}$ bei $800^{\circ}$ hergestellt ${ }^{19}$.

Die Nucleinsäuren- ${ }^{14} \mathrm{C}$ wurden aus 60 bzw. 220 Puppen ${ }^{*}$, denen $3-4$ Tage nach der Verpuppung Aminoimidazol-carboxyamid- $\left[4 \cdot{ }^{14} \mathrm{C}\right]$ bzw. Glycin- $\left[2 \cdot{ }^{14} \mathrm{C}\right]$ gespritzt worden war, gewonnen, indem die Puppen 48 bzw. 72 Stdn. nach Applikation der markierten Verbindungen getötet und in Anlehnung an $\mathrm{W}_{\mathrm{ACKER}}{ }^{20}$ die Nucleinsäuren isoliert wurden. Die Entfernung von Eiweißresten erfolgte nicht durch Octanol und Chloroform, sondern die Nucleinsäuren wurden, gelöst in 0,14-m. Kochsalzlösung, durch eine mit Sephadex G 50 gefüllte Säule gegeben. Die Radioaktivitäts-Ausbeute betrug 2-3 Prozent. Die isolierten Nucleinsäuren wurden zur Hydrolyse in 0,1-n. Natronlauge 4,5 Stdn. unter Rückfluß erhitzt. Die Abtrennung von Desoxyribonucleinsäure (DNS) erfolgte durch Sephadex G50. Nach Neutralisation des RNS-Hydrolysats wurde eingeengt und ein Teil auf eine Dowex-1 Säule gegeben, nach Waschen mit Wasser wurde Cytidylsäure und Adenylsäure mit 0,002-n. Salzsäure und Uridyl- und Guanylsäure mit 0,003-n. Salzsäure eluiert ${ }^{21}$. Mit Hilfe der bekannten UV-Extinktionen wurde die Menge und die spez. Radioaktivität bestimmt. Im Falle des Glycinversuches ergaben sich folgende Werte:

Guanylsäure 1,61 mg Aktivität $3,18 \cdot 10^{7} \mathrm{ipm} / \mathrm{mMol}$, Adenylsäure $2,28 \mathrm{mg}$ Aktivität $4,25 \cdot 10^{7} \mathrm{ipm} / \mathrm{mMol}$, Uridylsäure $1,1 \mathrm{mg}$ Aktivität $4,47 \cdot 10^{6} \mathrm{ipm} / \mathrm{mMol}$.

14 T. W. Goodwin u. A. A. Horton, Nature [London] 191, 772 [1961].

15 S. Aaronson u. E. Rodriguez. J. Bacteriol. 75, 660 [1958].

16 E. Vieira u. E. Shaw, J. biol. Chemistry 236, 2507 [1961].

17 J. J. Reynolds u. G. M. Brown, J. biol. Chemistry 237, PC 2713 [1962].

18 A. Murray u. D. Williams, Organic Syntheses with Isotopes, Part I S. 396.

19 O. I. Andrejetwa u. G. U. Kostikowa, Conference on the Use of Radioisotopes in the Physical Sciences and Industry, Kopenhagen, 6. - 17. Sept. 1960.

* Herrn Prof. Dr. E. Bünning und Frl. Dr. G. Joenrens, Tübingen, danken wir wiederum für die regelmäßige Zusendung von Kohlweißlingsgelegen.

20 A. Wacker u. D. Hartmans, Z. Naturforschg. 12 b, 181 [1957].

21 W. E. Cонn, J. Amer. chem. Soc. 72, 1471, 2811 [1950]. 
Die von der Sephadexsäule erhaltene DNS-Lösung wurde eingeengt. Zur Charakterisierung wurde das UVSpektrum aufgenommen und die D is c h e - Reaktion ${ }^{22}$ ausgeführt.

Das Mengenverhältnis DNS zu RNS war ca. $1: 6$ und das Radioaktivitätsverhältnis ca. $1: 8$.

Die Synthese von Pterin- $\left[2-{ }^{14} \mathrm{C}\right]$ erfolgte im $30-\mathrm{mg}$ Maßstab durch Kondensation von 2.4.5-Triamino-6-hydroxypyrimidin- $\left[2{ }^{14} \mathrm{C}\right]$ mit der Natriumbisulfit-Verbindung des Glyoxals ${ }^{23}$. Nach Reinigung über eine Aluminiumoxydsäule $(2 \cdot 30 \mathrm{~cm}$, Elutionsmittel Methanol: 2-n. Ammoniak = $70: 30$ ) und Umfällen betrug die Ausbeute 9,8 mg (44\% d. Th.).

Das 2.4-Diamino-5-formylamino-6-hydroxypyrimidin$\left[2-{ }^{14} \mathrm{C}\right]$ wurde nach Pfleiderer ${ }^{24}$ im 40-mg-Maßstab mit $76 \%$ Ausbeute dargestellt. Folsäure- $\left[2-{ }^{14} \mathrm{C}\right]$ und 2.4.5-Triamino-6-hydroxy-pyrimidin- $\left[2-{ }^{14} \mathrm{C}\right]$ wurden auf den von uns früher beschriebenen Wegen gewonnen ${ }^{25}$. Zur Darstellung von Pterin-8-carbonsäure- $\left[2-{ }^{14} \mathrm{C}\right]$ und Pterin-8-aldehyd-[2-14 C] wurde von 8-Tetrahydroxybutylpterin- $\left[2-{ }^{14} \mathrm{C}\right]$ ausgegangen, das nach WEYGAND et al. hergestellt worden war ${ }^{26}$.

\section{Applikation dermarkierten Verbin-} dungen und A u a r beitung

Die Menge der pro Puppe applizierten Verbindungen betrug im allgemeinen $25-50 \mu \mathrm{g}$. Die Applikation der markierten Verbindungen, die Isolierung, Reinigung und der Abbau des Leukopterins erfolgte wie beschrieben ${ }^{4}$. Die Trennung von Leukopterin, Isoxanthopterin und anderen, in sehr geringen Mengen vorhandenen Pterinen wurde jedoch, falls es von Interesse war, durch Aufgaben der alkalischen Pterinlösung auf eine Aluminiumoxydsäule bewirkt. Beim Entwickeln mit konz. Ammoniaklösung bleibt lediglich das Leukopterin am Start zurück. Diese Schicht wurde in heißer $2-n$. $\mathrm{NaOH}$ extrahiert, worauf die Lösung in 10-proz. heiße Salzsäure gegossen wurde.

Alle Radioaktivitäts-Messungen erfolgten im Gaszählrohr ${ }^{27}$. Bei der Aufarbeitung auf Purine ${ }^{4}$ wurde neben Guanin noch Harnsäure, Xanthin, Hypoxanthin, 2.8-Dioxyadenin, Isoguanin und Adenin isoliert. Das bisher in der Natur nur selten aufgefundene 2.8-Dioxyadenin wurde durch Vergleich mit einem synthetischen Produkt identifiziert ${ }^{28}$. Die übrigen Purine wurden auf Grund ihres chromatographischen und uv-spektroskopischen Verhaltens erkannt.

\section{Ergebnisse und Diskussion}

In Tab. 1 sind die Ergebnisse, die beim Vergleich der spez. Radioaktivitäten von Guanin und Adenin

22 Z. Dische, in: The Nucleic Acids Bd. I S. 294, Academic Press Inc., Publ., New York 1955.

23 C. K. Cain, M. F. Malette u. E. C. Taylor, Jr., J. Amer. chem. Soc. 68, 1996 [1946].

24 W. Pfleiderer, Chem. Ber. 90, 2272 [1957].

25 F. Weygand, H. J. Mann u. H. Simon, Chem. Ber. 85, 463 [1952]. mit Leukopterin nach verschiedenen Applikationszeiten von Glycin- $\left[2-{ }^{14} \mathrm{C}\right]$ erhalten wurden, zusammengestellt.

Im Falle der Vorpuppen verweilte das angebotene Glycin ca. 10 Tage im Organismus, im Falle der am 7. Tag gespritzten Puppen war die Verweilzeit nur ca. $2-3$ Tage.

Das Verhältnis der spezifischen Radioaktivitäten von Guanin und Leukopterin ist nach Gabe von Glycin- $\left[2{ }^{14} \mathrm{C}\right]$ nahe 1 , unabhängig davon, ob das Glycin- $\left[2{ }^{14} \mathrm{C}\right] 10$ Tage oder $2-3$ Tage vor dem Schlüpfen der Schmetterlinge appliziert wird. Dies gilt sowohl für das Guanin, das aus den Körpern der Schmetterlinge isoliert wurde, als auch für das aus den Flügeln isolierte. Allerdings zeigt der Vergleich der spezifischen Radioaktivitäten von Guanin aus Körpern und Flügeln das Vorliegen verschiedener, nicht im Gleichgewicht befindlicher Guaninpools. Das Verhältnis Adenin/Leukopterin weicht stärker von $1 \mathrm{ab}$.

Die Einbaurate von Glycin in das Leukopterin steigt bei kürzerer Applikationszeit um den Faktor 2. Auch die Einbaurate in das Guanin ist bei kürzerer Verweildauer des Glycins höher. Besonders drastisch gilt das für das Adenin. Es ist deutlich zu sehen, daß auch relativ kurz vor dem Schlüpfen der Falter noch eine starke Purinbiogenese abläuft. Auf Grund der praktisch gleichen spezifischen Radioaktivitäten von Guanin und Leukopterin ist es völlig auszuschließen, daß Pterin und Purin unabhängig voneinander synthetisiert werden. Denn wenn das Guanin im Laufe der Entwicklung weniger wird ${ }^{4}$ und das Leukopterin mit der Zeit zunimmt, die Bildung beider jedoch nichts miteinander zu tun hat, kann keine Gleichheit der spezifischen Aktivitäten erreicht werden.

Wenn sich der Weg der Purin- und Pterin-Biogenese nicht verzweigt, wie schon durch die Ergebnisse mit Glycin wahrscheinlich gemacht wird, so sollte auch bei der Gabe eines unmittelbaren Purinvorläufers, wie Amino-imidazolcarboxamid, Gleichheit der spezifischen Radioaktivität erreicht werden. Die Ergebnisse der Versuche zeigt Tab. 2. Bei der

26 F. Weygand, A. Wacker u. V. Schmied-Kowarzik, Chem. Ber. 82, 25 [1949].

27 H. Simon, H. Daniel u. J. F. Klebe, Angew. Chem. 71, 303 [1959] ; H. Simon u. F. Berthold, Atomwirtschaft VII, 498 [1962].

28 A. Bendich, J. F. Tinker u. G. B. Brown, J. Amer. chem. Soc. 70, 3113 [1948] ; A. Bendich, G. B. Brown, F. S. Philips u. J. B. Tiersch, J. biol. Chemistry 183, 267 [1950]. 


\begin{tabular}{|c|c|c|c|c|c|c|}
\hline $\begin{array}{l}\text { Zeit der Applikation } \\
\text { und Herkunft des } \\
\text { isolierten Materials }\end{array}$ & \multicolumn{3}{|c|}{$\begin{array}{l}\text { Spez. Radioaktivität in } \\
\mathrm{ipm} / \mathrm{mMol} \cdot 10^{-6} \text { und Einbaurate }\end{array}$} & \multicolumn{3}{|c|}{$\begin{array}{l}\text { Verhältnisse der spez. } \\
\text { Radioaktivitäten }\end{array}$} \\
\hline Vorpuppen, Körper & Leukopterin & $\begin{array}{c}\text { Guanin } \\
6,70 \\
1: 153^{1}\end{array}$ & $\begin{array}{c}\text { Adenin } \\
\begin{array}{l}7,5 \\
1: 122\end{array}\end{array}$ & $\begin{array}{c}\text { Guanin: } \\
\text { Leukopterin } \\
1,3^{2}\end{array}$ & $\begin{array}{c}\text { Adenin: } \\
\text { Leukopterin } \\
1,5^{2}\end{array}$ & $\begin{array}{c}\text { Guanin: } \\
\text { Adenin } \\
0,89\end{array}$ \\
\hline Vorpuppen, Flügel & $\begin{array}{l}5,03 \\
1: 53^{1}\end{array}$ & 7,50 & 9,3 & 1,5 & 1,8 & 0,81 \\
\hline $\begin{array}{l}\text { 7. Tag nach Ver- } \\
\text { puppung. Körper }\end{array}$ & & $\begin{array}{c}7,66 \\
1: 135\end{array}$ & $\begin{array}{l}20,6 \\
1: 44\end{array}$ & $0,8^{2}$ & $2,1^{2}$ & 0,36 \\
\hline 7. Tag nach Ver- & 9,$5 ; 10,5$ & 12,8 & 16,7 & 1,3 & 1,7 & 0,78 \\
\hline puppung, Flügel & $1: 26$ & 13,4 & 17,0 & & & \\
\hline
\end{tabular}

Tab. 1. Vergleich der Einbauraten und der spez. Radioaktivitäten nach Gabe von Glycin- $\left[2 \cdot{ }^{14} \mathrm{C}\right]$. ${ }^{1}$ Diese Verhältnisse geben die Einbauraten wieder. Die Einbaurate ist der Bruchteil der Radioaktivität, den die isolierte Verbindung von der Radioaktivität des applizierten Vorläufers hat. ${ }^{2}$ Verglichen mit dem Leukopterin aus den Flügeln.

\begin{tabular}{|c|c|c|c|c|c|c|}
\hline \multirow{2}{*}{$\begin{array}{l}\text { Zeit der Applikation } \\
\text { [Tag nach Verpuppung] } \\
\text { und Herkunft des } \\
\text { isolierten Materials }\end{array}$} & \multicolumn{3}{|c|}{$\begin{array}{l}\text { Spez. Radioaktivität in } \\
\mathrm{ipm} / \mathrm{mMol} \cdot 10^{-6} \text { und Einbaurate }\end{array}$} & \multicolumn{3}{|c|}{$\begin{array}{l}\text { Verhältnisse der spez. } \\
\text { Radioaktivitäten }\end{array}$} \\
\hline & Leukopterin & Guanin & Adenin & $\begin{array}{l}\text { Guanin: } \\
\text { Leukopterin }\end{array}$ & $\begin{array}{l}\text { Adenin: } \\
\text { Leukopterin }\end{array}$ & $\begin{array}{l}\text { Guanin: } \\
\text { Adenin }\end{array}$ \\
\hline Vorpuppen & $\begin{array}{l}1,42 \\
1: 46^{1}\end{array}$ & $\begin{array}{c}1,88 \\
1: 134\end{array}$ & $\begin{array}{c}1,92 \\
1: 117\end{array}$ & 1,3 & 1,3 & 1,0 \\
\hline 2. Körper & & $\begin{array}{l}4,86 \\
1,52\end{array}$ & $\begin{array}{l}5,0 \\
1,45\end{array}$ & $2,8^{2}$ & $2,8^{2}$ & 0,97 \\
\hline 2. Flügel & $\begin{array}{l}1,76 \\
1: 37\end{array}$ & 1,28 & 2,35 & 0,7 & 1,3 & 0,54 \\
\hline $\begin{array}{l}\text { 6. Flügel für } \\
\text { Leukopterin, Körper } \\
\text { für Purine }\end{array}$ & $\begin{array}{l}3,65 \\
1: 18\end{array}$ & $\begin{array}{l}13,1 \\
1: 20\end{array}$ & $\begin{array}{l}18,9 \\
1: 12\end{array}$ & 3,6 & 5,2 & 0,69 \\
\hline 7. Körper & & $\begin{array}{l}12,6 \\
1: 21\end{array}$ & $\begin{array}{l}14,8 \\
1: 16\end{array}$ & $4.8^{2}$ & $5,7^{2}$ & 0,85 \\
\hline 7. Flügel & $\begin{array}{l}2,61 \\
1,25\end{array}$ & 2,77 & & 1,06 & & \\
\hline
\end{tabular}

Tab. 2. Vergleich der Einbauraten und der spez. Radioaktivitäten von Leukopterin, Guanin und Adenin nach Gabe von Aminoimidazolcarboxyamid-4- ${ }^{14} \mathrm{C}$. ${ }^{1}$ Diese Verhältnisse geben die Einbauraten wieder (vgl. Tab. 1). ${ }^{2}$ Verglichen mit dem Leukopterin aus den Flügeln.

Gabe an Vorpuppen ist das Verhältnis der spezifischen Radioaktivitäten von Pterin und Purin wieder nahe 1. Das nach Applikation am 2. Tag nach der Verpuppung sich ergebende Verhältnis ist schon fast 3, um dann zum 6. Tag auf 3,6 für Guanin und 5,2 für Adenin anzusteigen. Nach Applikation am 7. Tag zeigt sich schließlich ein Wert von nahe 5 für Guanin bzw. gegen 6 für Adenin. Dieser Befund unterscheidet sich vom Glycinversuch. Vergleicht man allerdings das Verhältnis Purin/Leukopterin in den Flügeln, so ist es wieder nahe 1 . Außerdem sieht man jetzt das Vorliegen getrennter Pools sehr deutlich. Unabhängig von der Applikationszeit ist die spez. Aktivität von Leukopterin und Guanin bzw. Adenin am gleichen Ort praktisch gleich. Hier fällt die starke Zunahme der Einbauraten in die Purine mit kürze- ren Verweilzeiten der applizierten Verbindungen im Organismus auf. Sie steigt zwischen Applikation in die Vorpuppen und Applikation in die Puppen am 7. Tag für Guanin um den Faktor 6,4, für Adenin sogar um 7,3, für Leukopterin dagegen beträgt die Zunahme nur knapp 2.

Dies ist ein Beweis dafür, daß die Pterinbildung über die Purinbildung verlaufen muß. Der Guaningehalt der Schmetterlinge beträgt nur noch die Hälfte von dem der Puppen ${ }^{4}$, d. h. man hat eine starke Abnahme der Absolutmenge, dagegen eine drastische Erhöhung der Einbaurate. Die Pterinmenge dagegen nimmt zweifellos im Verlauf der Umwandlung Puppe $\rightarrow$ Schmetterling sehr stark zu. Würde ein Biogeneseweg unter Umgehung der Purine existieren, so müßte die Zunahme der Ein- 


\begin{tabular}{|c|c|c|c|c|c|c|}
\hline & \multicolumn{6}{|c|}{ Spezifische Radioaktivität der Purine in ipm $/ \mathrm{mMol} \cdot 10^{-6}$} \\
\hline & Hypoxanthin & Xanthin & Guanin & Adenin & Isoguanin & $\begin{array}{l}\text { 2.8-Dioxy- } \\
\text { adenin }\end{array}$ \\
\hline $\begin{array}{l}\text { Körper } \\
\text { Flügel } \\
\text { ungefähre Menge im } \\
\text { gesamten Schmetter- } \\
\text { ling in } \mu \mathrm{g}\end{array}$ & $\begin{array}{l}19,8 \\
- \\
25\end{array}$ & $\begin{array}{l}10 \\
- \\
15\end{array}$ & $\begin{array}{r}12,6 \\
2,8 \\
110\end{array}$ & $\begin{array}{c}14,8 \\
-\end{array}$ & $\begin{array}{c}-\overline{2,9} \\
30-50\end{array}$ & $\begin{array}{c}7,2 \\
- \\
40-60\end{array}$ \\
\hline
\end{tabular}

Tab. 3. Vergleich der molaren Radioaktivitäten aller aus Schmetterlingen isolierten Purine nach Gabe von Amino-imidazolcarboxamid- $\left[4-{ }^{14} \mathrm{C}\right]$ am 7 . Tag nach der Verpuppung.

baurate des Pterins sehr viel höher sein als die der Purine, da gegen Schluß der Entwicklungszeit, zu der Pterin gebildet wird, der Vorläufer dahin abgezogen werden müßte, während der Fluß in die Purine, die mengenmäßig abnehmen, gering sein sollte. Wie jedoch die Einbauraten zeigen, wird auch noch gegen Schluß Purin gebildet und noch mehr allerdings wird in Pterin abgeschoben.

Tab. 3 zeigt die molaren Radioaktivitäten von allen aus den Schmetterlingen isolierten Purinen nach Gabe von Amino-imidazolcarboxyamid- [4- $\left.{ }^{14} \mathrm{C}\right]$ am 7. Tag nach der Verpuppung (vgl. auch Tab. 2) . Xanthin und Hypoxanthin wurden aus den Körpern isoliert, 2.8-Dioxyadenin war sowohl in den Körpern als auch in den Flügeln vorhanden, während Isoguanin in nennenswerten Mengen nur in den Flügeln gefunden wurde. Die ebenfalls vorhandene Harnsäure wurde nicht bestimmt. In diesem Entwicklungsstadium scheint prozentual mehr Hypoxanthin gebildet zu werden als vorher, da seine spez. Aktivität offensichtlich weniger durch bereits vorhandenes Material verdünnt wird, als die der anderen Purine. Die etwas zu niedrige Aktivität des Xanthins ist wahrscheinlich nicht signifikant. Seine Menge war sehr gering. Auf jeden Fall zeigen die Werte, daß die verschiedenen Purinpools auch gegen Schluß der Puppenruhe in den Körpern noch im Austausch miteinander stehen, sonst wäre bei den verschiedenen Poolgrößen die weitgehende Gleichheit der spez. Aktivitäten nicht zu erklären. Die Purine in den Flügeln sind dagegen schon nicht mehr mit denen des Körpers im Gleichgewicht.

In Tab. 4 sind die Ergebnisse der Verwertung von Nucleinsäuren als solche, Desoxyribonucleinsäure, Ribonucleinsäurehydrolysat sowie Adenylsäure und Guanylsäure wiedergegeben.

Mit Ausnahme der Desoxyribonucleinsäure werden alle angebotenen Verbindungen sehr gut für die Leukopterinbildung verwertet. Die Einbauraten sind für Nucleinsäuren ebenso gut bzw. noch besser als für Aminoimidazolcarboxamid, die für Guanyl- und Adenylsäure besser als für alle anderen bisher von uns angebotenen Verbindungen. Die höhere Einbaurate der Nucleinsäure aus Aminoimidazolcarboxamid gegenüber der aus Glycin- $\left[2-{ }^{14} \mathrm{C}\right]$ gewonnenen ist so zu erklären, daß die Nucleinsäuren

\begin{tabular}{|c|c|c|c|}
\hline $\begin{array}{c}\text { Zeit der } \\
\text { Applikation } \\
\text { [Tag nach } \\
\text { Verpuppung] }\end{array}$ & $\begin{array}{l}\text { Markierte } \\
\text { Verbindung }\end{array}$ & $\begin{array}{c}\text { applizierte } \\
\text { Radio- } \\
\text { aktivität } \\
\text { pro Falter } \\
\text { [ipm] }\end{array}$ & $\begin{array}{l}\text { Einbau- } \\
\text { rate }\end{array}$ \\
\hline 3. & $\begin{array}{l}\text { Nucleinsäuren aus } \\
\text { Aminoimid- } \\
\text { azolcarboxy- } \\
\text { amid-[4-14 }]\end{array}$ & 1860 & $1: 17$ \\
\hline 3. & $\begin{array}{l}\text { Nucleinsäure aus } \\
\text { Glycin- }\left[2-{ }^{14} \mathrm{C}\right]\end{array}$ & 10425 & $1: 27$ \\
\hline 6. & $\begin{array}{l}\text { Ribonucleinsäure- } \\
\text { Hydrolysat aus } \\
\text { Glycin- }[2-14 \mathrm{C}]\end{array}$ & 1385 & $1: 21$ \\
\hline 5. & $\begin{array}{l}\text { Desoxyribonuclein- } \\
\text { säure aus }\end{array}$ & 1.500 & $1: 21$ \\
\hline & Glycin-[2-14C] & 1360 & $1: 190$ \\
\hline 6. & $\begin{array}{l}\text { Adenylsäure aus } \\
\text { Glycin- }\left[2-{ }^{14} \mathrm{C}\right]\end{array}$ & 3030 & $1: 5,5$ \\
\hline 6. & $\begin{array}{c}\text { Guanylsäure aus } \\
\text { Glycin- }\left[2-{ }^{14} \mathrm{C}\right]\end{array}$ & 200 & $1: 11$ \\
\hline
\end{tabular}

Tab. 4. Einbauraten in das Leukopterin nach Applikation von markierten Nucleinsäuren, DNS, RNS-Hydrolysat sowie Adenylsäure und Guanylsäure.

aus Aminoimidazolcarboxamid- $\left[4-{ }^{14} \mathrm{C}\right]$ Radioaktivität ausschließlich in den Purinbasen besitzen dürften, während in den aus Glycin- $\left[2{ }^{-14} \mathrm{C}\right]$ gewonnenen ein gewisser Prozentsatz der Radioaktivität in den Pyrimidinen und in der Ribose enthalten sein wird. Der Grund für den geringeren Einbau von Guanylsäure, verglichen mit dem von Adenylsäure, ist nicht klar. Der Unterschied kann z. T. methodisch bedingt sein. Die Menge der zur Verfügung stehenden radioaktiven Guanylsäure war sehr gering. Andererseits fällt beim Vergleich der Einbauraten von Amino- 
imidazolcarboxamid und Glycin in Guanin und Adenin auf, daß der Adeninstoffwechsel wesentlich intensiver zu sein scheint. Die spez. Radioaktivität von Adenin ist stets etwas höher als die von Guanin. Die Einbauraten sind bei kurzen Applikationszeiten für Adenin sogar beträchtlich höher.

Die Verteilung der Radioaktivität zwischen C-8 und C-9 nach Gabe von Ribose-[ $\left[{ }^{14} \mathrm{C}\right]$ in Abhängigkeit von der Zeit zeigt Tab. 5. Die Zunahme der

\begin{tabular}{|c|c|c|c|c|}
\hline $\begin{array}{c}\text { Zeit der } \\
\text { Applikation } \\
\text { [Tage nach } \\
\text { Verpuppung] }\end{array}$ & $\begin{array}{l}\text { Einbau- } \\
\text { rate }^{1}\end{array}$ & $\begin{array}{c}\text { Radioa } \\
\text { C-2 } \\
{[\%]}\end{array}$ & $\begin{array}{c}\text { xtivität in } \\
\text { C-8 + C-9 } \\
{[\%]}\end{array}$ & $\begin{array}{l}\text { Verhältnis } \\
\text { C-8:C-9 }\end{array}$ \\
\hline 4 & $1: 91$ & 14 & 64 & $42: 58$ \\
\hline 5 & $1: 43$ & 10,2 & 54 & $30: 70$ \\
\hline 6 & $1: 25$ & 7,3 & 56 & $28: 72$ \\
\hline 7 & $1: 23$ & 7,2 & 69 & $16: 84$ \\
\hline 8 & $1: 22$ & 6,4 & 65 & $16: 84$ \\
\hline 9 & $1: 32$ & - & 76 & $14: 86$ \\
\hline
\end{tabular}

Tab. 5. Einbauraten in Leukopterin und Verhältnis der Aktivität von C-8 : C-9 nach Gabe von Ribose- $\left[1-{ }^{14} \mathrm{C}\right]$. ${ }^{1}$ Definition der Einbaurate s. Tab. 1.

Radioaktivität in C-9 ist mit kürzerer Verweilzeit im Organismus sehr ausgeprägt. Der ${ }^{14} \mathrm{C}$-Gehalt in C-2 des Leukopterins zeigt, daß auch noch gegen Ende der Entwicklung ein wesentlicher Prozentsatz der Ribose in den allgemeinen Stoffwechsel gerät und eine Verteilung C-8 : C-9 im Verhältnis 0 : 100 somit nicht erwartet werden kann. Die Versuche zeigen jedoch mit Sicherheit, daß C-9 von C-1 einer Pentose kommt. Durch Vergleich der Einbauraten in C-8 und C-9 nach Gabe von Glucose- $\left[1-{ }^{14} \mathrm{C}\right]$ und Glucose- $\left[2 \cdot{ }^{14} \mathrm{C}\right]$ haben wir bereits unwahrscheinlich machen können, daß C-8 und C-9 von einem C-2Baustein (aktiver Glykolaldehyd) stammt ${ }^{4}$.

Durch die geschilderten Befunde hat das von uns bereits vorgeschlagene Bildungsschema ${ }^{4}$ noch mehr an Wahrscheinlichkeit gewonnen.

Aus Guanosin oder einem Phosphat wird C-8 eliminiert und der Ribosylrest unter Ausbildung des Ringes vermutlich nach einer A m a d o r i - Umlagerung (II) direkt einkondensiert (III). Diese Kondensation erfolgt, wie Woop und Mitarb. ${ }^{29}$ gezeigt haben, spontan. Der Übergang zum Xanthopterin kann dann durch oxydative Spaltung oder Dealdolisierung und Dehydrierung aus IV erfolgen. Durch Xanthinoxydase erfolgt Übergang in das Leukopterin.

Durch Wasserabspaltung und Dehydrierung kann aus IV das 2-Amino-6-hydroxypteridin (V) entstehen, das dann zu Isoxanthopterin führt. Die Schlüsselverbindung III kann durch eine Aldolasereaktion austauschen, wodurch andere Pterine (III a) entstehen können. Der Austausch mit einem Einkohlenstoffbaustein könnte den Vorläufer für die Folsäure bilden ${ }^{30}$.

\begin{tabular}{|c|c|c|c|c|c|c|}
\hline \multirow{2}{*}{$\begin{array}{l}\text { Angebotene Substanz und } \\
\text { Zeit der Applikation } \\
\text { [Tag nach Verpuppung] }\end{array}$} & \multicolumn{3}{|c|}{$\begin{array}{l}\text { Spez. Radioaktivität in jpm/mMol } \\
\text { und Einbaurate }\end{array}$} & \multicolumn{3}{|c|}{$\begin{array}{l}\text { Verhältnisse der spez. } \\
\text { Radioaktivitäten }\end{array}$} \\
\hline & Leukopterin & Guanin & Adenin & $\begin{array}{c}\text { Guanin: } \\
\text { Leukopterin }\end{array}$ & $\begin{array}{c}\text { Adenin: } \\
\text { Leucopterin }\end{array}$ & $\begin{array}{l}\text { Guanin: } \\
\text { Adenin }\end{array}$ \\
\hline $\begin{array}{l}\text { Pterin- } 2-14 \mathrm{C} \\
4 . \\
2.4 .5 \text {-Triamino- } 6 \text {-hydroxy- } \\
\text { pyrimidin- }[2-14 \mathrm{C}] \\
4 . \\
2.4-\text {-Diamino-5-formyl-amino-6- } \\
\text { hydroxy-pyrimidin- }\left[2-{ }^{14} \mathrm{C}\right] \\
4 . \\
\text { Folsäure-[2-14C] } \\
\text { Pterin-8-carbonsäure- }[2-14 \mathrm{C}] \\
\text { Pterin-8-aldehyd-[2-14C] } \\
8-\text { Tetrahydroxybutyl-pterin- } \\
{[2-14 \mathrm{C}]}\end{array}$ & $\begin{array}{l}\left.1: 355^{\mathrm{a}}\right) \\
\\
1,54 \cdot 10^{5} \\
1: 240 \\
1,62 \cdot 10^{4} \\
1: 12000 \\
\left.1: 39^{\mathrm{c}}\right) \\
1: 1000 \\
1: 252 \\
1: 2000\end{array}$ & $\begin{array}{l}\left.1,42 \cdot 10^{6 b}\right) \\
1: 540\end{array}$ & $\begin{array}{l}1,41 \cdot 10^{4} \\
1: 6400\end{array}$ & 87,5 & 11,6 & 11.4 \\
\hline
\end{tabular}

Tab. 6. Einbauraten verschiedener ${ }^{14} \mathrm{C}$-markierter Substanzen in Leukopterin, Guanin und Adenin. a) Vor Abtrennung des Isoxanthopterins ergab sich eine scheinbare Einbaurate von 1 : 10. b) Sehr wahrscheinlich ist bei der Aufarbeitung Guanin entstanden. c) Leukopterin und Isoxanthopterin wurden nicht getrennt.

29 H. C. Wood u. A. Stuart, Proc. Chem. Soc. 1962, 151 [1962] ; H. C. Wood u. T. Neilon, J. chem. Soc. [London] 1962, 44 [1962].
30 Vgl. L. Jaenicke u. P. C. Chan, Angew. Chem. 72, 752 [1960]. 
Wenn wir für diesen Mechanismus auch noch keine direkten Beweise haben, so sprechen doch folgende Befunde neben den bereits erwähnten noch dafür:

Das 2-Amino-6-hydroxypteridin (V) kommt in den Schmetterlingen vor. Es wird, wenn überhaupt nur in sehr geringem $\mathrm{Ma} \beta$ in Leukopterin umgewandelt (Tab.6). Dagegen erfolgt eine Umwandlung in Isoxanthopterin. Interessanterweise findet sich auch eine geringe aber signifikante Radioaktivität im Guanin (Tab. 6). Danach kann man sogar eine Umkehr des Überganges Purin $\rightarrow$ Pterin annehmen. Ein solcher Übergang wurde kürzlich auch von McNutr ${ }^{31}$ gefunden.

Das 2.4.5-Triamino-6-hydroxy-pyrimidin wird, wenn auch nur schwach, zur Leukopterinbildung verwendet (Tab.6). Der geringe Einbau kann darauf beruhen, daß seine Glykosidierung keine physiologische Reaktion ist und daß das empfindliche Diamin daher schneller irgendwelchen Oxydationsreaktionen anheim fällt. Dies entspräche den chemischen Befunden, wonach 2.4.5-Triamino-6-hydroxy-pyrimidin nicht an der 4-ständigen Aminogruppe glykosidiert werden kann und als freie Base sehr wenig beständig ist.

Kürzlich berichteten BaUgH und SHAW $^{32}$ über eine 85-proz. spezifische Einbaurate von 2.4.5-Triamino-6hydroxypyrimidin in Pterine durch Cornynebacterium $s p .{ }^{*}$. Die Autoren berichteten weiter, da $\beta$ mit einer spezifischen Einbaurate von $0,23 \%$ aus Guanin $-{ }^{14} \mathrm{C}$ 2.4.5-Triamino-6-hydroxypyrimidin gebildet wird.

Erstaunlich ist, daß das 2.4-Diamino-5-formylamino-6-hydroxypyrimidin überhaupt nicht verwertet wird (Tab. 6). Das läßt den Gedanken aufkommen, daß C-8 nicht durch hydrolytische Öffnung des



31 W. S. McNutr, Pterinsymposium Stuttgart Sept. 1962.

* Seltsamerweise berichten die Autoren nicht, wie sie von den spontan in der Nährlösung entstehenden Pterinen abtrennen. Das Nährmedium enthält soviel Glucose, daß sich nach unseren Erfahrungen schon beim Stehen des Me- diums mit dem zugesetzten 2.4.5-Triamino-6-hydroxy-pyrimidin bei Zimmertemperatur große Mengen Pterine bilden.

32 C. M. Baugh u. E. Shaw, Biochem. biophysic. Res. Commun. 10, 28 [1963]. 
Imidazolringes und durch Abspaltung von Ameisensäure eliminiert wird, sondern in einer anderen Weise. Man könnte daran denken, daß in 8-Stellung zunächst oxydiert wird. Das aufgefundene 2.8-Dioxyadenin könnte in diese Richtung weisen. Ferner ist bekannt, daß Adenin durch Xanthinoxydase über 8-Hydroxyadenin zu 2.8-Dihydroxyadenin oxydiert werden kann.

Auf der Suche nach einem weiteren Zwischenprodukt erhielten wir papierchromatographisch aus Puppen, denen $40 \mathrm{Stdn}$. vor dem Abtöten ${ }^{14} \mathrm{C}$-markierte Ribose appliziert worden war, eine fluoreszierende Substanz, die bei Perjodatoxydation radioaktiven Formaldehyd, Ameisensäure und Pterinaldehyd liefert.

Für unseren Mechanismus sprechen weiterhin das Auffinden von 2-Amino-6-hydroxy-8-trihydroxypropyl-pteridin mit einer Phosphatgruppe in 3 '-Stellung durch Gото und Forrest ${ }^{33}$ und die Ergebnisse von Reynolds und Brown ${ }^{17}$, wonach zellfreie Ex-

${ }^{33}$ M. Goto u. H. S. Forrest, Biophys. biochem. Res. Commun. 6, 180 [1961]. trakte von Escherichia coli Guanosin, Guanylsäure bzw. Guanosinphosphate in Dihydrofolsäure überführen. Guanin und verschiedene Ribosephosphate werden schlechter verwertet.

Von den weiteren von uns angebotenen Pterinen mit Substituenten in 8-Stellung zeigt nur die Folsäure einen sehr guten Einbau in das Leukopterin bzw. Isoxanthopterin. Beide Pterine wurden zur Analyse nicht getrennt. Dies entspräche den Befunden von BlaIR ${ }^{34}$. Pterin-8-aldehyd- $\left[2{ }^{14} \mathrm{C}\right]$ wird nur gering verwertet. Praktisch kein Einbau erfolgt durch D-arabo-8-(Tetra-hydroxybutyl)-peterin-[2$\left.{ }^{14} \mathrm{C}\right]$ und Pterin-8-carbonsäure- $\left[2-{ }^{14} \mathrm{C}\right]$. Dies zeigt, daß diese Verbindungen nicht auf dem Weg zum Leucopterin liegen.

Der Deutschen Forschungsgemeinschaft und dem $B$ undesministerium für wissen schaftliche Forschung danken wir für finanzielle Unterstützung, Frl. H. Pflaumer für zahlreiche, sorgfältig ausgeführte ${ }^{14} \mathrm{C}$-Analysen.

34 J. A. Blair, Nature [London] 192, 757 [1961]. 